\title{
Gravitational dual of averaged free CFT's over the Narain lattice
}

\author{
Alfredo Pérez and Ricardo Troncoso \\ Centro de Estudios Científicos (CECs), \\ Avenida Arturo Prat 514, Valdivia, Chile \\ E-mail: aperez@cecs.cl, troncoso@cecs.cl
}

ABSTRACT: It has been recently argued that the averaging of free CFT's over the Narain lattice can be holographically described through a Chern-Simons theory for $\mathrm{U}(1)^{D} \times \mathrm{U}(1)^{D}$ with a precise prescription to sum over three-dimensional handlebodies.

We show that a gravitational dual of these averaged CFT's would be provided by Einstein gravity on $\mathrm{AdS}_{3}$ with $\mathrm{U}(1)^{D-1} \times \mathrm{U}(1)^{D-1}$ gauge fields, endowed with a precise set of boundary conditions closely related to the "soft hairy" ones. Gravitational excitations then go along diagonal SL $(2, \mathbb{R})$ generators, so that the asymptotic symmetries are spanned by $\mathrm{U}(1)^{D} \times \mathrm{U}(1)^{D}$ currents. The stress-energy tensor can then be geometrically seen as composite of these currents through a twisted Sugawara construction. Our boundary conditions are such that for the reduced phase space, there is a one-to-one map between the configurations in the gravitational and the purely abelian theories. The partition function in the bulk could then also be performed either from a non-abelian Chern-Simons theory for two copies of SL $(2, \mathbb{R}) \times U(1)^{D-1}$ generators, or formally through a path integral along the family of allowed configurations for the metric. The new boundary conditions naturally accommodate BTZ black holes, and the microscopic number of states then appears to be manifestly positive and suitably accounted for from the partition function in the bulk. The inclusion of higher spin currents through an extended twisted Sugawara construction in the context of higher spin gravity is also briefly addressed.

Keywords: Black Holes, Classical Theories of Gravity, Gauge-gravity correspondence

ARXIV EPRINT: 2006.08216 


\section{Contents}

1 Introduction 1

2 Gravitational dual and its boundary conditions 3

3 Geometrical emergence of composite Virasoro generators 4

4 Contact with the abelian theory in the bulk $\quad 6$

5 Metric formalism, black holes and microscopic counting of states $\quad 7$

6 Extended Sugawara construction and boundary conditions for higher spin

gravity 8

\section{Introduction}

It has been recently shown that the path integral of Jackiw-Teitelboim gravity [1-4] can be seen as the dual of an average over an ensemble of theories at the boundary, instead of a single specific one [5]. Aiming to lift this result to higher dimensions, in refs. [6, 7], the bulk dual of averaged free two-dimensional CFT's over the Narain lattice [8,9] has been identified as an abelian $\mathrm{U}(1)^{D} \times \mathrm{U}(1)^{D}$ Chern-Simons theory, given by

$$
I=\sum_{I=1}^{D} \int_{Y}\left(A_{I}^{+} d A_{I}^{+}-A_{I}^{-} d A_{I}^{-}\right),
$$

with a precise prescription to sum over three-dimensional oriented handlebodies $Y$ with fixed boundary $\partial Y=\Sigma$. The partition function then fulfills

$$
\sum_{Y} Z_{Y}^{\mathrm{U}(1)^{2 D}}(\tau)=\left\langle Z_{\Sigma}(m, \tau)\right\rangle
$$

where $Z_{\Sigma}(m, \tau)$ stands for the partition function of the free $\mathrm{CFT}_{2}$ at a point $m$ of the lattice, and $\tau$ collectively denotes the modular parameters of the Riemann surface $\Sigma$. The bracket $\langle\cdots\rangle$ stands for averaging over Narain moduli space, whose measure is determined by the Zamolodchikov metric, normalized so that the volume of Narain moduli space is equal to one.

The result can be transparently visualized for a single connected surface $\Sigma$ of genus 1 . In this case, the partition function of the free $\mathrm{CFT}_{2}$ is given by

$$
Z_{\Sigma}(m, \tau)=\frac{\Theta(m, \tau)}{|\eta(\tau)|^{2 D}}
$$


where $\eta(\tau)$ is the Dedekind eta function, and $\Theta(m, \tau)$ is the "Siegel-Narain" theta function. Therefore, by virtue of the Siegel-Weil formula [10-13] the right-hand side of eq. (1.2) reads

$$
\left\langle Z_{\Sigma}(m, \tau)\right\rangle=\frac{E_{D / 2}(\tau)}{\operatorname{Im}(\tau)^{D / 2}|\eta(\tau)|^{2 D}},
$$

where $E_{D / 2}$ is the real analytic (non-holomorphic) modular invariant Eisenstein series.

Following [6,7], the left-hand side of eq. (1.2) can be obtained as follows. Although the abelian Chern-Simons theory carries no metric in the bulk, the perturbative contribution to the partition function can be evaluated on Euclidean (thermal) $\mathrm{AdS}_{3}$, whose boundary is a torus with modular parameter $\tau$. The 1-loop partition function is then given by that of $D$ left- and right-moving chiral bosons, determined by the vacuum character of the $\mathrm{U}(1)^{D} \times \mathrm{U}(1)^{D}$ current algebra [14], i.e.,

$$
Z_{\mathrm{EAdS}_{3}}^{\mathrm{U}(1)^{2 D}}(\tau, \bar{\tau})=\frac{1}{\eta(\tau)^{D} \eta(-\bar{\tau})^{D}}=\chi_{0}(\tau) \bar{\chi}_{0}(\bar{\tau})
$$

For our purposes, it is worth highlighting that this is a direct consequence of the fact that the asymptotic symmetries of the theory in the bulk are described by the $\mathrm{U}(1)^{D} \times \mathrm{U}(1)^{D}$ affine algebra.

The full partition function in the bulk, $Z_{\text {bulk }}(\tau)$, can then be obtained once the sum over handlebodies is carried out. This is explicitly performed along the lines of [15], i.e., summing over all modular images in SL $(2, \mathbb{Z})$ quotiented by those preserving Euclidean $\mathrm{AdS}_{3}$, given by $\Gamma$, being spanned by $T$-modular transformations. The full partition function can then be expressed as

$$
Z_{\text {bulk }}(\tau)=\sum_{\gamma \in \operatorname{SL}(2, \mathbb{Z}) / \Gamma} \frac{1}{|\eta(\gamma \tau)|^{2 D}}=\frac{1}{\operatorname{Im}(\tau)^{D / 2}|\eta(\tau)|^{2 D}} \sum_{\gamma \in \operatorname{SL}(2, \mathbb{Z}) / \Gamma} \operatorname{Im}(\gamma \tau)^{D / 2},
$$

which by virtue of the definition of the Eisenstein series,

$$
E_{s}(\tau)=\sum_{\gamma \in \operatorname{SL}(2, \mathbb{Z}) / \Gamma} \operatorname{Im}(\gamma \tau)^{s}
$$

agrees with the average of dual theories at the boundary, $Z_{\text {bulk }}(\tau)=\left\langle Z_{\Sigma}(m, \tau)\right\rangle$.

As pointed out in [6], since the theory in the bulk is abelian, it possesses "boundary photons" instead of boundary gravitons, that are described by chiral and anti-chiral current algebras at the boundary. Nonetheless, a composite boundary graviton could always emerge through the Sugawara construction, and it is then natural to wonder about its gravitational dual in the bulk.

One of the main purposes of our work is showing that a simple setup to realize the gravitational dual in the bulk can be given by Einstein gravity on $\mathrm{AdS}_{3}$ supplemented by $2(D-1)$ abelian gauge fields, endowed with a precise choice of boundary conditions, being closely related to those in $[16,17]$. Our boundary conditions guarantee that the asymptotic symmetries are spanned by the $\mathrm{U}(1)^{D} \times \mathrm{U}(1)^{D}$ affine algebra, so that in the reduced phase space there is a one-to-one correspondence between configurations in the gravitational and abelian theories. 


\section{Gravitational dual and its boundary conditions}

Let us consider Einstein gravity on $\mathrm{AdS}_{3}$ with an additional set of $2(D-1)$ abelian gauge fields, so that the action can be written as

$$
I\left[g_{\mu \nu}, A_{\mu I}^{+}, A_{\mu I}^{-}\right]=\frac{1}{16 \pi G} \int_{Y} d^{3} x \sqrt{-g}\left(R+2 l^{-2}\right)+\sum_{I=2}^{D} \int_{Y}\left(A_{I}^{+} d A_{I}^{+}-A_{I}^{-} d A_{I}^{-}\right) .
$$

It is useful to express the gravitational sector as the difference of two Chern-Simons actions for $\operatorname{SL}(2, \mathbb{R})$ with level $k=l /(4 G)[18,19]$, so that the non-abelian gauge fields relate to the dreibein and the spin connection according to ${ }^{1}$

$$
A_{s l(2, \mathbb{R})}^{ \pm}=\omega \pm \frac{e}{l} .
$$

The action (2.1) can be written as the difference of two Chern-Simons actions for $\mathrm{SL}(2, \mathbb{R}) \times \mathrm{U}(1)^{D-1}$, up to a boundary term. Following [20] and also [21, 22], it is useful to express the $\mathrm{SL}(2, \mathbb{R}) \times \mathrm{U}(1)^{D-1}$ gauge fields $A^{ \pm}$in the asymptotic region as

$$
A^{ \pm}=b_{ \pm}^{-1}\left(d+a^{ \pm}\right) b_{ \pm}
$$

where the dependence on the radial coordinate is completely captured by the group elements $b_{ \pm}=b_{ \pm}(r)$. The asymptotic behavior becomes then fully determined once the excitations of the auxiliary gauge fields $a^{ \pm}$are specified in the asymptotic region.

We propose a set of boundary conditions, being such that gravitational excitations go along the diagonal $s l(2, \mathbb{R})$ generators $L_{0}$, so that

$$
a^{ \pm}=\left(\mathcal{J}^{ \pm} L_{0}+\sum_{I=2}^{D} \mathcal{J}_{I}^{ \pm} Z_{I}\right) d x^{ \pm}
$$

where $x^{ \pm}=\frac{t}{l} \pm \phi$, and $Z_{I}$ stand for the $\mathrm{U}(1)$ generators. Note that the field equations, $F=0$, imply that left and right excitations are chiral and anti-chiral, respectively.

The asymptotic form of the gauge fields is preserved under gauge transformations $\delta_{\lambda} a^{ \pm}=d \lambda^{ \pm}+\left[a^{ \pm}, \lambda^{ \pm}\right]$, with $\lambda^{ \pm}=\eta_{i}^{ \pm} L_{i}+\eta_{I}^{ \pm} Z_{I}$, provided that the excitations transform according to

$$
\delta \mathcal{J}^{ \pm}=\partial_{ \pm} \eta^{ \pm}, \quad \delta \mathcal{J}_{I}^{ \pm}=\partial_{ \pm} \eta_{I}^{ \pm}
$$

where the parameters $\eta_{I}^{ \pm}$and $\eta^{ \pm}:=\eta_{0}^{ \pm}$are (anti-)chiral. Additional gauge transformations spanned by suitable parameters $\eta_{1}^{ \pm}$and $\eta_{-1}^{ \pm}$can be seen to be trivial proper gauge transformations that do not contribute to the canonical generators. The conserved charges can be obtained following different approaches [23, 24], so that they are given by $Q=Q^{+}-Q^{-}$, with

$$
Q^{ \pm}\left[\eta^{ \pm}, \eta_{I}^{ \pm}\right]=\mp \int d \phi\left(\frac{k}{4 \pi} \eta^{ \pm} \mathcal{J}^{ \pm}+\sum_{I=2}^{D} 2 \eta_{I}^{ \pm} \mathcal{J}_{I}^{ \pm}\right)
$$

\footnotetext{
${ }^{1}$ The $\operatorname{sl}(2, \mathbb{R})$ generators are normalized according to $\left[L_{i}, L_{j}\right]=(i-j) L_{i+j}$, with $i, j=-1,0,1$, so that the nonvanishing components of the invariant bilinear form are given by $\left\langle L_{1}, L_{-1}\right\rangle=-1$ and $\left\langle L_{0}, L_{0}\right\rangle=\frac{1}{2}$.
} 
The asymptotic symmetry algebra spanned by the generators (2.5) can then either be obtained from the computation of their Dirac brackets, or more quickly, by virtue of $\delta_{\chi} Q[\epsilon]=\{Q[\epsilon], Q[\chi]\}$, from the transformation law of the dynamical fields in (2.4). It is readily found to be that of $\mathrm{U}(1)^{D} \times \mathrm{U}(1)^{D}$ currents. Indeed, expanding in Fourier modes according to

$$
\mathcal{J}^{ \pm}=\frac{2}{\sqrt{k}} \sum_{n} J_{n}^{(1) \pm} e^{ \pm i n \phi}, \quad \mathcal{J}_{I}^{ \pm}=\frac{1}{\sqrt{2 \pi}} \sum_{n} J_{n}^{(I) \pm} e^{ \pm i n \phi}
$$

the nonvanishing brackets read

$$
i\left\{J_{n}^{(I) \pm}, J_{m}^{(K) \pm}\right\}=\frac{1}{2} n \delta^{I K} \delta_{m+n, 0},
$$

where for the modes, the indices $I, K$ take values on $1,2, \ldots, D$.

Our boundary conditions could be regarded as an extension of the "soft hairy" ones in $[16,17]$ for which the chemical potentials are allowed to depend on the dynamical fields in a precise way. Different sets of boundary conditions whose chemical potentials also depend on the dynamical fields have been devised in order to make contact with two-dimensional integrable systems in refs. [25-29].

\section{Geometrical emergence of composite Virasoro generators}

Following the lines of $[16,17]$, a twisted Sugawara construction naturally emerges from the comparison of the asymptotic structure of spacetime for our boundary conditions with that of Brown-Henneaux [30] endowed with $\mathrm{U}(1)^{D-1} \times \mathrm{U}(1)^{D-1}$ currents. Thus, the asymptotic symmetry algebra of the standard boundary conditions (with spectral flow), spanned by two copies of the semidirect sum of Virasoro with the $\mathrm{U}(1)^{D-1}$ affine algebra, arises from composites of the $\mathrm{U}(1)^{D} \times \mathrm{U}(1)^{D}$ currents.

In order to perform the comparison one has to express both sets of boundary conditions in terms of the same variables. Note that for our asymptotic behavior, described through eqs. (2.2) and (2.3), the gravitational auxiliary gauge fields $a_{s l(2, \mathbb{R})}^{ \pm}$are written in the diagonal gauge, while for the standard Brown-Henneaux boundary conditions they are expressed in the highest weight gauge [20]. Therefore, the searched for relationships can be obtained once the asymptotic form of our connections in (2.2) and (2.3) are written in the highest weight gauge for a suitable generic choice of some still unspecified chemical potentials as in [21, 22].

For simplicity, we carry out the comparison just for the "+" copy of the gauge fields. Indeed, one proceeds in the same way for the other copy, and so we drop the superscript "+".

The asymptotic form of the gravitational part of the connection in the highest weight gauge reads

$$
\hat{A}=\hat{b}^{-1}(d+\hat{a}) \hat{b}
$$


with $\hat{b}=e^{\rho L_{0}}$, and

$$
\begin{aligned}
& \hat{a}_{\phi}=L_{1}-\frac{1}{2}\left(\mathcal{L}-\frac{4 \pi}{k} \sum_{I=2}^{D} \mathcal{J}_{I}^{2}\right) L_{-1} \\
& l \hat{a}_{t}=\mu L_{1}-\mu^{\prime} L_{0}+\frac{1}{2}\left[\mu^{\prime \prime}-\left(\mathcal{L}-\frac{4 \pi}{k} \sum_{I=2}^{D} \mathcal{J}_{I}^{2}\right) \mu\right] L_{-1},
\end{aligned}
$$

where $\mathcal{L}$ and $\mu$ stand for arbitrary functions of $t$ and $\phi$, and prime denotes derivatives with respect to $\phi$. Note that the spectral flow was already incorporated in the auxiliary connections, see e.g. [31].

One can then show that the auxiliary gauge field $\hat{a}$ in the highest weight gauge and $a$ in the diagonal gauge are related by a group element $g$ that is permissible in the sense of [22], i.e., it does not interfere with the asymptotic structure. Indeed, $\hat{a}=g^{-1}(d+a) g$ with

$$
g=\exp \left(x L_{1}\right) \exp \left(-\frac{1}{2} \mathcal{J} L_{-1}\right)
$$

where $x=x(t, \phi)$ fulfills $\mu=l \dot{x}-\mathcal{J} x$, and $x^{\prime}-\mathcal{J} x=1$. Consistency with the fact that $\mathcal{J}$ is a chiral mover implies that

$$
\mu^{\prime}+\mathcal{J}(1-\mu)=0
$$

which means that the chemical potential $\mu$ at the boundary is generically given by

$$
\mu(t, \phi)=1+f(t) \exp \left[\int_{0}^{\phi} \mathcal{J}\left(\phi^{\prime}\right) d \phi^{\prime}\right]
$$

where $f(t)$ is an arbitrary function of time.

The gauge fields $a$ and $\hat{a}$ are then mapped into each other provided that the boundary gravitons are related to the "boundary photons" through a twisted Sugawara construction

$$
\mathcal{L}=\frac{4 \pi}{k} \sum_{I=2}^{D} \mathcal{J}_{I}^{2}+\frac{1}{2} \mathcal{J}^{2}+\partial_{+} \mathcal{J}
$$

which in modes reads

$$
L_{n}=\sum_{I=2}^{D}\left(\sum_{p} J_{n-p}^{(I)} J_{p}^{(I)}\right)+\sum_{p} J_{n-p}^{(1)} J_{p}^{(1)}+i n \sqrt{k} J_{n}^{(1)} .
$$

Therefore, by virtue of (2.6) the modes $L_{n}$ fulfill the Virasoro algebra with the BrownHenneaux central charge

$$
i\left\{L_{n}, L_{m}\right\}=(n-m) L_{n+m}+\frac{1}{2} k n^{2} \delta_{n+m, 0},
$$

together with

$$
i\left\{L_{n}, J_{m}^{(I)}\right\}=-m J_{m+n}^{(I)}
$$

for $I=2, \ldots, D$. 
Note that the map between highest weight and diagonal gauge choices implies that the corresponding parameters $\hat{\eta}$, that preserve the asymptotic structure in the highest weight gauge, become field dependent. Indeed, under the action of an asymptotic symmetry spanned by a purely gravitational U (1) current, the parameters in the highest weight gauge fulfill

$$
\mathcal{J} \hat{\eta}-\hat{\eta}^{\prime}=\eta
$$

so that the transformation law of the boundary gravitons, $\delta \mathcal{L}=2 \mathcal{L} \partial_{+} \hat{\eta}+\hat{\eta} \partial_{+} \mathcal{L}-\partial_{+}^{3} \hat{\eta}$, is recovered from that of the boundary photons $\delta \mathcal{J}=\partial_{+} \eta$ by virtue of the twisted Sugawara relation (3.2).

It should be emphasized that, although the currents $L_{n}$ satisfy the Virasoro algebra, the relationship between the parameters in (3.3) implies that their associated generators $Q[\hat{\eta}]$ actually span the gravitational $\mathrm{U}(1)$ current algebra, because

$$
\delta Q=-\frac{k}{4 \pi} \int d \phi \hat{\eta} \delta \mathcal{L}=-\frac{k}{4 \pi} \int d \phi \eta \delta \mathcal{J} .
$$

As an ending remark of this section, we point out that since the chemical potentials $\mu^{ \pm}$are related to the lapse and shift functions of the boundary metric, they should be single valued. Therefore, eq. (3.1) implies that if the function $f(t)$ were chosen to be nonvanishing, the zero mode of $\mathcal{J}$ should be excluded. Nonetheless, for the choice $f(t)=0$ $(\mu=1)$, the zero modes of the $\mathrm{U}(1)$ currents are allowed.

\section{Contact with the abelian theory in the bulk}

The new boundary conditions ensure that the reduced phase space of the gravitational action (2.1) coincides with that of the abelian theory in (1.1). Therefore it is possible to establish a one-to-one map between configurations in the abelian theory and those of the gravitational one with our boundary conditions. The map is precisely given by

$$
A_{\mathrm{U}(1)^{D}}=h^{-1}\left(d+A_{\mathrm{SL}(2, \mathbb{R}) \times \mathrm{U}(1)^{D-1}}\right) h,
$$

with $h=b^{-1} b_{\mathrm{U}(1)^{D}}$, where $b=b(r)$ stands for the gauge group element in (2.2), and $b_{\mathrm{U}(1)^{D}}=b_{\mathrm{U}(1)^{D}}(r)$ corresponds to that of abelian theory. For instance, if the asymptotic form of the metric were given in normal coordinates, then the gravitational factor of the group element can be chosen as $b_{ \pm}^{s l(2, \mathbb{R})}(r)=\exp \left( \pm \frac{r}{2 l}\left(L_{1}-L_{-1}\right)\right)$.

One then concludes that the reduced phase space of the gravitational theory endowed with our boundary conditions becomes identical to that of the abelian theory; and therefore, performing the quantization of the reduced phase of both theories turns out to be equivalent.

It is worth pointing out that the partition function in the bulk could then be performed either from the non-abelian Chern-Simons theory, or formally through a path integral along a family of allowed configurations for the metric, i.e.

$$
\left\langle Z_{\Sigma}(m, \tau)\right\rangle=\sum_{Y} Z_{Y}^{\mathrm{U}(1)^{2 D}}(\tau)=\sum_{Y} Z_{Y}^{\left(\mathrm{SL}(2, \mathbb{R}) \times \mathrm{U}(1)^{D-1}\right)^{2}}(\tau)=\sum_{Y} \int \mathcal{D} g_{\mu \nu} \prod_{I=2}^{D} \mathcal{D} A_{\mu I} e^{-I_{E}}
$$


where $I_{E}$ stands for the Euclidean continuation of the action (2.1). Note that the FadeevPopov determinant of the gravitational theory should be carefully determined so that it precisely captures the relevant contributions from gauge fixing that are compatible with our boundary conditions.

It should also be emphasized that the presence of a metric in the bulk endows $Y$ with a well-defined notion of (Riemannian) manifold with boundary $\Sigma$.

Our one-to-one map between gravitational and abelian configurations has been devised to work in a precise way when $\Sigma$ has the topology of a torus. It would be interesting to explore how our results could be extended for surfaces $\Sigma$ of higher genus.

\section{Metric formalism, black holes and microscopic counting of states}

Note that the abelian fields in the gravitational theory (2.1) are described through a ChernSimons action, so that they do not couple to the metric; and hence, the spacetime geometry solves the Einstein equations with negative cosmological constant in vacuum. For our choice of boundary conditions $(2.2),(2.3)$, the most general solution is given by

$$
d s^{2}=d r^{2}+\frac{l^{2}}{4}\left(\mathcal{J}_{+}^{2} d x^{+2}+\mathcal{J}_{-}^{2} d x^{-2}-2 \cosh \left(\frac{2 r}{l}\right) \mathcal{J}_{+} \mathcal{J}_{-} d x^{+} d x^{-}\right)
$$

where $\mathcal{J}^{ \pm}=\mathcal{J}^{ \pm}\left(x^{ \pm}\right)$are (anti-)chiral.

Diffeomorphisms that preserve the form of the metric, $\delta_{\xi} g_{\mu \nu}=\mathcal{L}_{\xi} g_{\mu \nu}$, are then spanned by $\xi=\xi^{\mu} \partial_{\mu}$, with $\xi^{r}=0$ and

$$
\xi^{+}=\frac{\eta^{+}}{\mathcal{J}_{+}}, \quad \xi^{-}=\frac{\eta^{-}}{\mathcal{J}_{-}},
$$

provided that $\delta \mathcal{J}_{ \pm}=\partial_{ \pm} \eta^{ \pm}$, with $\partial_{\mp} \eta^{ \pm}=0$. The canonical generators associated to this set of diffeomorphisms fulfill the abelian current algebra.

It is reassuring to verify that the metric and the asymptotic symmetries agree with those obtained from the Chern-Simons formulation with $g_{\mu \nu}=\frac{l^{2}}{2}\left\langle\left(A_{\mu}^{+}-A_{\mu}^{-}\right)\left(A_{\nu}^{+}-A_{\nu}^{-}\right)\right\rangle$, and $\eta^{ \pm} L_{0}=\xi^{\mu} a_{\mu s l(2, \mathbb{R})}^{ \pm}$.

Note that generic BTZ black holes [32, 33] belong to the reduced phase space, as it can be seen from the zero modes of (5.1). Anti-de Sitter spacetime is not in the (Lorentzian) spectrum, but it is clearly brought back in the Euclidean continuation. It is worth highlighting that massless and extremal BTZ black holes become automatically excluded from the allowed configurations, since the metric degenerates for vanishing $J_{0}^{(1)+}$ or $J_{0}^{(1)-}$. Indeed, excluding the latter configurations turns out to be natural in the Euclidean continuation, since they possess a different topology that includes an additional boundary.

From the abelian and non-abelian Chern-Simons approaches, excluding zero modes for the chiral fields appears to be reasonably justified. Nevertheless, making contact with the bulk metric brings in a puzzling feature, because zero modes precisely correspond to BTZ black holes which cannot be so naturally excluded from the spectrum. In fact, their Bekenstein-Hawking entropy, once expressed in terms of the global charges reads

$$
S=\frac{A}{4 G}=2 \pi \sqrt{k}\left(J_{0}^{(1)+}+J_{0}^{(1)-}\right),
$$


in agreement with [16, 17], and by virtue of (3.2) is equivalent to the Cardy formula supplemented by the spectral flow, i.e.,

$$
S=\sqrt{2} \pi k\left(\sqrt{\mathcal{L}^{+}-\frac{4 \pi}{k} \sum_{I=2}^{D}\left(\mathcal{J}_{I}^{+}\right)^{2}}+\sqrt{\mathcal{L}^{-}-\frac{4 \pi}{k} \sum_{I=2}^{D}\left(\mathcal{J}_{I}^{-}\right)^{2}}\right)
$$

Interestingly, the full microscopic number of states appears to be manifestly positive and suitably accounted for from the partition function in the bulk (1.4), being equivalent to averaging over moduli space at the boundary (1.3). Indeed, as pointed out in [7], the partition function can also be expressed as

$$
Z_{\mathrm{bulk}}=\sum_{\ell=-\infty}^{\infty} \int_{|\ell|}^{\infty} d \Delta \rho_{\ell}(\Delta) \chi_{(\Delta+\ell) / 2}(\tau) \bar{\chi}_{(\Delta-\ell) / 2}(\bar{\tau})
$$

where

$$
\rho_{\ell}(\Delta)=\frac{2 \pi^{D} \sigma_{1-D}(\ell)}{\Gamma(D / 2)^{2} \zeta(D)}\left(\Delta^{2}-\ell^{2}\right)^{D / 2-1}
$$

is the density of non-vacuum primary states of spin $\ell=\Delta^{+}-\Delta^{-}$and scaling dimension $\Delta=\Delta^{+}+\Delta^{-}[10]$, with

$$
\Delta^{ \pm}=\frac{k}{2}\left(\mathcal{L}^{ \pm}-\frac{4 \pi}{k} \sum_{I=2}^{D}\left(\mathcal{J}_{I}^{ \pm}\right)^{2}+\frac{1}{2}\right)
$$

and $\sigma_{1-D}(\ell)$ stands for the sum of $m^{1-D}$ for all positive integers $m$ dividing $\ell$.

It should be highlighted that $\rho_{\ell}(\Delta)$ is manifestly positive and it only corresponds to the density of primaries. Therefore, once descendants are also included in the counting, the total number of states grows according to the Cardy formula. ${ }^{2}$ In fact, the asymptotic growth of the number of states is clearly dominated by the contribution coming from the $\mathrm{U}(1)$ characters, while according to $\rho_{\ell}(\Delta)$, subleading contributions necessarily involve left and right movers, so that the full partition function does not holomorphically factorizes.

\section{Extended Sugawara construction and boundary conditions for higher spin gravity}

One of the key points to obtain the gravitational dual of the Sugawara formula was the inclusion of a suitable twisting, precisely as in the analogue of the Miura map in (3.2), so that the stress-energy tensor fulfills the Virasoro algebra with the Brown-Henneaux central charge. The precise twisting is geometrically realized in the bulk for the gravitational theory (2.1) with our boundary conditions.

\footnotetext{
${ }^{2}$ We thank an anonymous referee for pointing out this remark.
} 
Due to the presence of the additional $U^{2(D-1)}$ fields, it is natural to wonder about a similar construction that allowed to incorporate higher spin currents. Indeed, for $D>2$, the expected extended Sugawara construction can be obtained along the lines of [34], so that

$$
\begin{aligned}
\mathcal{L} & =\frac{k}{4 \pi}\left(\frac{2}{3} \mathcal{J}_{(3)}^{2}+\frac{1}{2} \mathcal{J}^{2}+\partial_{+} \mathcal{J}\right) \\
\mathcal{W} & =-\frac{k}{6 \pi}\left(-\frac{8}{9} \mathcal{J}_{(3)}^{3}+2 \mathcal{J}^{2} \mathcal{J}_{(3)}+\mathcal{J}_{(3)} \partial_{+} \mathcal{J}+3 \mathcal{J} \partial_{+} \mathcal{J}_{(3)}+\partial_{+}^{2} \mathcal{J}_{(3)}\right),
\end{aligned}
$$

precisely fulfill the $W_{3}$ algebra with the corresponding central extension obtained from gravity with a spin three field for the boundary conditions in $[35,36]$. In this case, the extended Sugawara construction can be seen to emerge from higher spin gravity with $\mathrm{SL}(3, \mathbb{R}) \times \mathrm{SL}(3, \mathbb{R})$ gauge group (see e.g. [37-39]), endowed with $\mathrm{U}(1)^{D-2} \times \mathrm{U}(1)^{D-2}$ gauge fields.

Thus, in a generic case, the bulk dual can be given by higher spin gravity for $\mathrm{SL}(N, \mathbb{R}) \times \mathrm{SL}(N, \mathbb{R})$ with $\mathrm{U}(1)^{D-N+1} \times \mathrm{U}(1)^{D-N+1}$ gauge fields, with boundary conditions defined through $(2.2)$, so that the auxiliary gauge fields in the asymptotic region are given by

$$
a^{ \pm}=\left(\mathcal{J}^{ \pm} L_{0}+\sum_{s=3}^{N} \mathcal{J}_{(s)}^{ \pm} W_{0}^{(s)}+\sum_{I=N}^{D} \mathcal{J}_{I}^{ \pm} Z_{I}\right) d x^{ \pm},
$$

where $L_{0}$ and $W_{0}^{(s)}$ are the generators of the Cartan subalgebra of SL $(N, \mathbb{R})$. Therefore, the asymptotic symmetry algebra can be readily seen to be spanned by the $\mathrm{U}(1)^{D} \times \mathrm{U}(1)^{D}$ current algebra.

The geometrical realization of the extended Sugawara construction then naturally emerges from the comparison of our boundary conditions in the diagonal gauge with those in the highest weight gauge $[21,22,35,36]$.

\section{Acknowledgments}

We thank Fábio Novaes for useful discussions. This research has been partially supported by Fondecyt grants № 1171162, 1181496, 1181031. The Centro de Estudios Científicos (CECs) is funded by the Chilean Government through the Centers of Excellence Base Financing Program of Conicyt.

Open Access. This article is distributed under the terms of the Creative Commons Attribution License (CC-BY 4.0), which permits any use, distribution and reproduction in any medium, provided the original author(s) and source are credited.

\section{References}

[1] C. Teitelboim, Gravitation and Hamiltonian Structure in Two Space-Time Dimensions, Phys. Lett. B 126 (1983) 41 [INSPIRE].

[2] R. Jackiw, Liouville field theory: A two-dimensional model for gravity?, in Quantum Theory Of Gravity, S. Christensen ed., Adam Hilger, Bristol, pp. 403-420 (1984) [InSPIRE]. 
[3] C. Teitelboim, The Hamiltonian structure of two-dimensional space-time and its relation with the conformal anomaly, in Quantum Theory Of Gravity, S. Christensen ed., Adam Hilger, Bristol, pp. 327-344 (1984) [INSPIRE].

[4] R. Jackiw, Lower Dimensional Gravity, Nucl. Phys. B 252 (1985) 343 [inSPIRE].

[5] P. Saad, S.H. Shenker and D. Stanford, JT gravity as a matrix integral, arXiv:1903.11115 [INSPIRE].

[6] A. Maloney and E. Witten, Averaging Over Narain Moduli Space, arXiv:2006.04855 [INSPIRE].

[7] N. Afkhami-Jeddi, H. Cohn, T. Hartman and A. Tajdini, Free partition functions and an averaged holographic duality, arXiv:2006.04839 [INSPIRE].

[8] K.S. Narain, New Heterotic String Theories in Uncompactified Dimensions < 10, Phys. Lett. $B 169$ (1986) 41 [INSPIRE].

[9] K.S. Narain, M.H. Sarmadi and E. Witten, A Note on Toroidal Compactification of Heterotic String Theory, Nucl. Phys. B 279 (1987) 369 [InSPIRE].

[10] C.L. Siegel, Indefinite quadratische formen und funktionentheorie I, Math. Ann. 124 (1951) 17.

[11] H. Maass and T. Srinivasan, Lectures on Siegels modular functions, Tata Institute of Fundamental Research (1955).

[12] A. Weil, Sur certains groupes d'opérateurs unitaires, Acta Math. 111 (1964) 143.

[13] A. Weil, Sur la formule de siegel dans la théorie des groupes classiques, Acta Math. 113 (1965) 1.

[14] M. Porrati and C. Yu, Kac-Moody and Virasoro Characters from the Perturbative Chern-Simons Path Integral, JHEP 05 (2019) 083 [arXiv: 1903.05100] [InSPIRE].

[15] A. Maloney and E. Witten, Quantum Gravity Partition Functions in Three Dimensions, JHEP 02 (2010) 029 [arXiv:0712.0155] [INSPIRE].

[16] H. Afshar et al., Soft Heisenberg hair on black holes in three dimensions, Phys. Rev. D 93 (2016) 101503 [arXiv: 1603.04824] [INSPIRE].

[17] H. Afshar, D. Grumiller, W. Merbis, A. Perez, D. Tempo and R. Troncoso, Soft hairy horizons in three spacetime dimensions, Phys. Rev. D 95 (2017) 106005 [arXiv:1611.09783] [INSPIRE].

[18] A. Achucarro and P.K. Townsend, A Chern-Simons Action for Three-Dimensional anti-de Sitter Supergravity Theories, Phys. Lett. B 180 (1986) 89 [InSPIRE].

[19] E. Witten, (2+1)-Dimensional Gravity as an Exactly Soluble System, Nucl. Phys. B 311 (1988) 46 [INSPIRE].

[20] O. Coussaert, M. Henneaux and P. van Driel, The Asymptotic dynamics of three-dimensional Einstein gravity with a negative cosmological constant, Class. Quant. Grav. 12 (1995) 2961 [gr-qc/9506019] [INSPIRE].

[21] M. Henneaux, A. Perez, D. Tempo and R. Troncoso, Chemical potentials in three-dimensional higher spin anti-de Sitter gravity, JHEP 12 (2013) 048 [arXiv:1309.4362] [INSPIRE].

[22] C. Bunster, M. Henneaux, A. Perez, D. Tempo and R. Troncoso, Generalized Black Holes in Three-dimensional Spacetime, JHEP 05 (2014) 031 [arXiv:1404.3305] [INSPIRE]. 
[23] T. Regge and C. Teitelboim, Role of Surface Integrals in the Hamiltonian Formulation of General Relativity, Annals Phys. 88 (1974) 286 [INSPIRE].

[24] G. Barnich and F. Brandt, Covariant theory of asymptotic symmetries, conservation laws and central charges, Nucl. Phys. B 633 (2002) 3 [hep-th/0111246] [INSPIRE].

[25] A. Pérez, D. Tempo and R. Troncoso, Boundary conditions for General Relativity on AdS 3 and the KdV hierarchy, JHEP 06 (2016) 103 [arXiv: 1605.04490] [INSPIRE].

[26] O. Fuentealba et al., Integrable systems with $\mathrm{BMS}_{3}$ Poisson structure and the dynamics of locally flat spacetimes, JHEP 01 (2018) 148 [arXiv:1711.02646] [INSPIRE].

[27] D. Melnikov, F. Novaes, A. Pérez and R. Troncoso, Lifshitz Scaling, Microstate Counting from Number Theory and Black Hole Entropy, JHEP 06 (2019) 054 [arXiv: 1808.04034] [INSPIRE].

[28] E. Ojeda and A. Pérez, Boundary conditions for General Relativity in three-dimensional spacetimes, integrable systems and the KdV/mKdV hierarchies, JHEP 08 (2019) 079 [arXiv: 1906.11226] [INSPIRE].

[29] D. Grumiller and W. Merbis, Near horizon dynamics of three dimensional black holes, SciPost Phys. 8 (2020) 010 [arXiv: 1906.10694] [INSPIRE].

[30] J. Brown and M. Henneaux, Central Charges in the Canonical Realization of Asymptotic Symmetries: An Example from Three-Dimensional Gravity, Commun. Math. Phys. 104 (1986) 207 [INSPIRE].

[31] M. Henneaux, L. Maoz and A. Schwimmer, Asymptotic dynamics and asymptotic symmetries of three-dimensional extended AdS supergravity, Annals Phys. 282 (2000) 31 [hep-th/9910013] [INSPIRE].

[32] M. Bañados, C. Teitelboim and J. Zanelli, The Black hole in three-dimensional space-time, Phys. Rev. Lett. 69 (1992) 1849 [hep-th/9204099] [inSPIRE].

[33] M. Bañados, M. Henneaux, C. Teitelboim and J. Zanelli, Geometry of the $(2+1)$ black hole, Phys. Rev. D 48 (1993) 1506 [Erratum ibid. 88 (2013) 069902] [gr-qc/9302012] [INSPIRE].

[34] D. Grumiller, A. Perez, S. Prohazka, D. Tempo and R. Troncoso, Higher Spin Black Holes with Soft Hair, JHEP 10 (2016) 119 [arXiv:1607.05360] [InSPIRE].

[35] M. Henneaux and S.-J. Rey, Nonlinear $W_{\infty}$ as Asymptotic Symmetry of Three-Dimensional Higher Spin Anti-de Sitter Gravity, JHEP 12 (2010) 007 [arXiv:1008.4579] [INSPIRE].

[36] A. Campoleoni, S. Fredenhagen, S. Pfenninger and S. Theisen, Asymptotic symmetries of three-dimensional gravity coupled to higher-spin fields, JHEP 11 (2010) 007 [arXiv: 1008.4744] [INSPIRE].

[37] M.P. Blencowe, A Consistent Interacting Massless Higher Spin Field Theory in D $=(2+1)$, Class. Quant. Grav. 6 (1989) 443 [inSPIRE].

[38] E. Bergshoeff, M.P. Blencowe and K.S. Stelle, Area Preserving Diffeomorphisms and Higher Spin Algebra, Commun. Math. Phys. 128 (1990) 213 [INSPIRE].

[39] M.A. Vasiliev, Higher spin gauge theories in four-dimensions, three-dimensions, and two-dimensions, Int. J. Mod. Phys. D 5 (1996) 763 [hep-th/9611024] [INSPIRE]. 